\title{
NR5A1 is required for functional maturation of Sertoli cells during postnatal development
}

\author{
Tomoko Kato ${ }^{1}$, Michiyo Esaki ${ }^{1}$, Ayami Matsuzawa ${ }^{1}$ and Yayoi Ikeda ${ }^{1,2}$ \\ ${ }^{1}$ Department of Histology and Cell Biology, Yokohama City University School of Medicine, Yokohama 236-0004, \\ Japan and ${ }^{2}$ Department of Anatomy, School of Dentistry, Aichi Gakuin University, Nagoya 464-8650, Japan
}

Correspondence should be addressed to Y Ikeda at Department of Anatomy, School of Dentistry, Aichi Gakuin University; Email: yayoi@dpc.agu.ac.jp

\begin{abstract}
The orphan nuclear receptor steroidogenic factor 1 (NR5A1 (SF-1)) is expressed in both Sertoli and Leydig cells in the testes. This study investigates the postnatal development of the testes of a gonad-specific Nr5a1 knockout (KO) mouse, in which Nr5a1 was specifically inactivated. The KO testes appeared histologically normal from postnatal day 0 (P0) until P7. However, disorganized germ cells, vacuoles, and giant cells appeared by P14 in the seminiferous tubules of KO but not control mice. Expression of NR5A1 and various factors was examined by immunohistochemistry (IHC). The number of NR5A1-positive Sertoli cells in the KO testes was lower compared with controls at all the developmental stages and decreased to nearly undetectable levels by P21. IHC for anti-Müllerian hormone and p27, immature and mature Sertoli cell markers, respectively, indicated a delay in Sertoli cell maturation in the KO testes. The number of Sertoli cell-expressing factors involved in Sertoli cell differentiation including WT1, SOX9, GATA4, and androgen receptor were lower in the KO testes compared with controls. Furthermore, fewer proliferating cell nuclear antigen-positive proliferative germ cells were observed, and the number of TUNEL-labeled cells was significantly higher in the KO testes compared with controls at P14 and P21, indicating impaired spermatogenesis. IHC for CYP11A1 (SCC) indicated the presence of steroidogenic Leydig cells in the interstitium of the KO testes at all stages examined. These results suggest that NR5A1 is essential for Sertoli cell maturation and therefore spermatogenesis, during postnatal testis development.

Reproduction (2012) 143 663-672
\end{abstract}

\section{Introduction}

The orphan nuclear receptor steroidogenic factor 1 (SF-1, officially designated NR5A1) was originally found as a transcription regulator of P450 cytochrome steroidogenic enzymes. Mammalian NR5A1 is expressed throughout development in steroidogenic and nonsteroidogenic cells in limited tissues along the reproductive axis, including the gonads, adrenals, pituitary, and the ventromedial hypothalamus. In the testes, Nr5a1 is expressed in two cell types, Sertoli and Leydig (lkeda et al. 1994). In vitro studies have suggested that NR5A1 functions in Sertoli cells within a regulatory complex that also contain transcription factors including $W T 1$, SOX9, and GATA4 to upregulate anti-Müllerian hormone $(A m h)$, the key gene for mammalian male sex differentiation (Shen et al. 1994, De Santa Barbara et al. 1998, Nachtigal et al. 1998, Arango et al. 1999, Watanabe et al. 2000). In Leydig cells, NR5A1 controls the expression of steroid biosynthetic enzyme genes including StAR protein (Star), cytochrome P450 cholesterol side-chain cleavage enzyme (Cyp11a1 (ScC)), 33-hydroxysteroid dehydrogenase $(H s d 3 b 4(3 \beta-H s d))$, and P450c17
(Ikeda et al. 1993). Mice homozygous for a null allele of Nr5a1 (original Nr5a1 knockout (KO)) exhibit abnormalities at multiple levels along the reproductive axis, including a lack of gonads and adrenals, reduction of pituitary gonadotropin production, and defective organization of the ventromedial hypothalamus, suggesting essential roles for NR5A1 in these tissues (Luo et al. 1994, Shen et al. 1994, Ikeda et al. 1995). The original Nr5a1 KO mice survive embryonic development but die soon after birth due to adrenal agenesis and a deficiency in adrenal corticoids. Owing to the neonatal death, it has been difficult to analyze any postnatal functions of NR5A1 using these animals. Furthermore, the gonads of the original $\mathrm{Nr} 5 \mathrm{a} 1 \mathrm{KO}$ mice degenerate by apoptosis just before differentiation at approximately embryonic day 10.5 (E10.5; Luo et al. 1994). Therefore, although it has been suggested that NR5A1 is required for the survival and proliferation of cells in the undifferentiated gonadal primordium, little is known about the role of NR5A1 after the initiation of testicular differentiation (Parker et al. 2002, Schimmer \& White 2010).

To circumvent these problems, conditional $\mathrm{KO}$ mice, in which Nr5a1 was specifically inactivated in the 
gonads using Cre-loxP recombination with Amhr2-Cre, were generated. The gonad-specific $\mathrm{Nr} 5 \mathrm{a} 1 \mathrm{KO}$ mice survive adulthood, and previous studies have demonstrated that the testes and ovaries are affected (Jeyasuria et al. 2004, Pelusi et al. 2008). In the previous study on the gonad-specific Nr5a1 KO testes, no Leydig cells were detected in the interstitium but Sertoli cells were detected within the testicular cord during embryonic development (Jeyasuria et al. 2004). Nonetheless, histological examination demonstrated markedly hypoplastic seminiferous tubules with no mature sperm by adulthood. As functional maturation of the Sertoli cells, occurring during postnatal development, is thought to be important for spermatogenesis (Sharpe et al. 2003), it is possible that abnormal development of the Sertoli cells results in the impaired spermatogenesis seen in the adult gonad-specific Nr5a1 KO testes. In conditional androgen receptor $(A r) \mathrm{KO}$ mice, which were also generated using the Amhr2-Cre, not only Leydig cells but also Sertoli cells are affected in the postnatal testes (Xu et al. 2007). Furthermore, Amhr2-Cre-mediated conditional $\mathrm{KO}$ of Gata4, which is expressed in both Sertoli and Leydig cells in embryonic development through adulthood, has been reported to display only Sertoli cell-specific ablation of GATA4 in postnatal testes (Kyrönlahti et al. 2011). These reports support the hypothesis that healthy Sertoli cells are required for spermatogenesis, and when Nr5a1 is ablated in Sertoli cells in the testes of gonad-specific Nr5a1 KO mice, their function is impaired leading to spermatogenesis defects.

To clarify the molecular defects of gonad-specific Nr5a1 KO testes, we characterized the postnatal development of the testes from P0 to P21, paying particular attention to the Sertoli cells and their fate.

\section{Results}

As described in the Materials and methods section, we used $\mathrm{Nr} 5 \mathrm{ar} 1^{\text {flox/- }}$, Amhr2 $2^{\text {cre/+ }}$ mice for the gonadspecific Nr5a1 KO in this study. For controls, we assessed

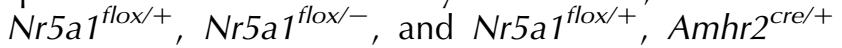
littermates at each time point. We examined the histology and immunoreactivity (ir) for NR5A1, and other markers of Sertoli and Leydig cells (as described below), and no differences were observed between these three groups (data not shown). Therefore, the data presented as controls are from mice of any one of these three genotypes.

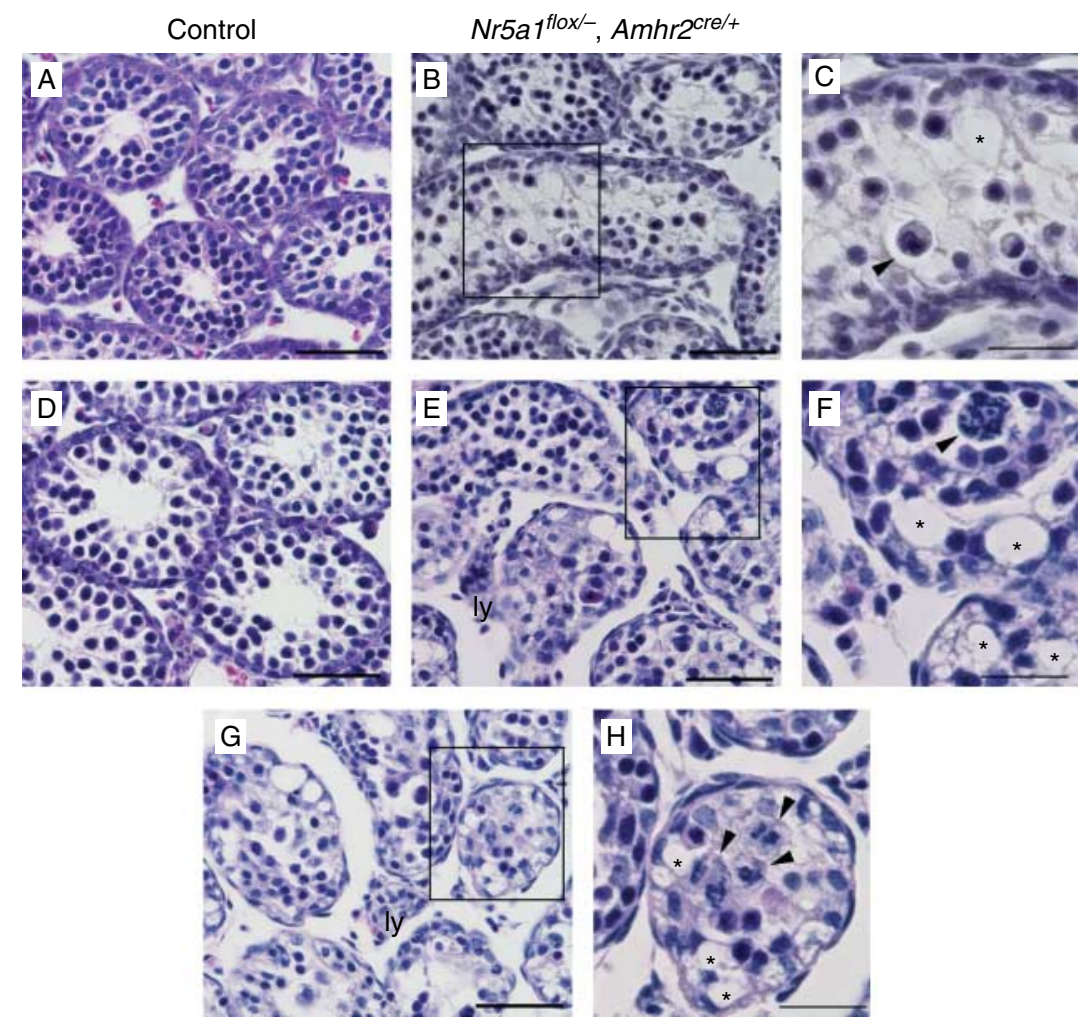

Figure 1 Histology of the gonad-specific Nr5a1 KO testes at P14 and P21. Representative photomicrographs of testes from controls (A and D) and Nr5a $1^{\text {flox } /-}$, Amhr2 ${ }^{\text {cre/+ }}$ (B, C, E, F, G and H) mice stained with H\&E. (A, B and C) P14. (D, E, F, G and H) P21. C, F and H are higher magnification views of areas within rectangles in $B, E$ and $G$ respectively. Asterisks and arrowheads indicate representative vacuoles and giant cells respectively. ly, Leydig cell clusters. Scale bars $=50 \mu \mathrm{m}$ in A, B, D, E and G and $25 \mu \mathrm{m}$ in C, F and $\mathrm{H}$. 


\section{Abnormal tubular architecture in the gonad-specific Nr5a1 KO mouse testis during prepubertal development}

We first examined the testicular histology at several time points from P0 to P21. At P0 and P7, there was no apparent difference in the architecture between the $\mathrm{KO}$ and control mouse testes (data not shown). However, abnormal morphology was observed in the $\mathrm{KO}$ mouse testes from P14 onward. At P14 and P21, the seminiferous tubules of the control mice consisted of layers of germ cells and Sertoli cells that were localized in the peripheral layer, with a luminal opening in the center (Fig. 1 A and D). In the KO mouse testes, however, the germ cells were irregularly arranged with fewer Sertoli cells compared with controls, vacuoles appeared, and many of the seminiferous tubules exhibited no lumen at P14 (Fig. 1B and C). At P21, the vacuoles and the depletion of Sertoli cells were more readily observed (Fig. 1E, F, G and H). Giant cells, which were never observed in control testes, appeared occasionally at P14 but abundantly at P21, within the tubule of the $\mathrm{KO}$ testis (Fig. 1C, F and $\mathrm{H}$, arrowheads). Leydig cells were detected in the interstitium of both control and $\mathrm{KO}$ testes at all the stages examined.

\section{Expression of NR5A1 in the gonad-specific Nr5a1 KO testis during postnatal development}

We next investigated testicular Nr5a1 expression by immunohistochemistry (IHC) at various stages during postnatal development. In control testes, Nr5a1 expression in the Sertoli cells was detected at all stages (Fig. 2A, C, E and G). In the gonad-specific Nr5a1 KO mouse testes, NR5A1-positive Sertoli cells were detected at stages P0 (Fig. 2B), P7 (Fig. 2D), and P14 (Fig. 2F) but were barely detectable at P21 (Fig. $2 \mathrm{H}$ ). To compare the number of NR5A1-positive Sertoli cells between $\mathrm{KO}$ and control mouse testes, we performed a positive cell count and showed that the number of NR5A1-positive Sertoli cells in the KOs was significantly lower than that in the controls at all stages examined (Fig. 2l).

\section{Impaired Sertoli cell development in the gonad-specific Nr5a1 KO testis during postnatal development}

To evaluate Sertoli cell maturation, which occurs during postnatal development, we examined the expression of p27 and AMH as markers for differentiated (Beumer et al. 1999) and immature (Rey 1998, Xu et al. 2007) Sertoli cells, respectively, using IHC. We found that at P14, p27positive Sertoli cells were lined along the basal region of the tubules in control testes (Fig. 3A), whereas the number of p27-positive Sertoli cells in each tubule was markedly reduced in the gonad-specific Nr5a1 KO mouse testes compared with that in the control testes (Fig. 3B). AMH ir was intense in most tubules at P0 and P7
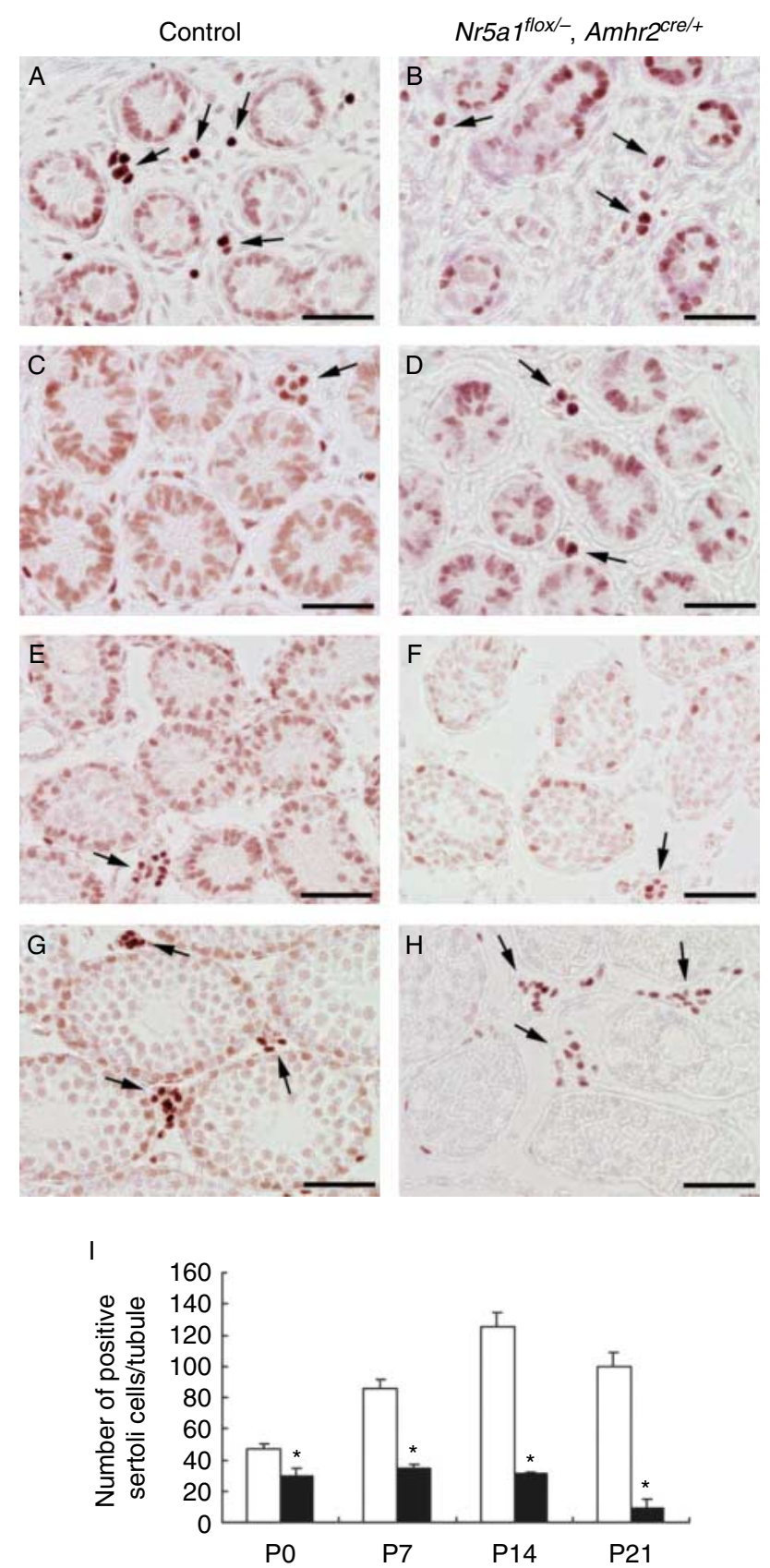

Figure $2 \mathrm{Nr} 5 \mathrm{a} 1$ expression in gonad-specific $\mathrm{Nr} 5 \mathrm{a} 1 \mathrm{KO}$ testes during postnatal development. Sections of testes from controls (left panels) and $\mathrm{Nr} 5 \mathrm{ar} 1^{\text {flox } /-}, \mathrm{Amhr} 2^{\text {cre/+ }}$ (right panels) mice at P0 (A and B), P7 (C and D), P14 (E and F), and P21 (G and H) were subjected to immunohistochemistry for NR5A1. Arrows indicate representative NR5A1-positive interstitial cells. Scale bars $=50 \mu \mathrm{m}$. (I) NR5A1positive Sertoli cells per tubule were counted. The relative number of control (open column, $n=3$ ) and Nr5a $1^{\text {flox } /-}$, Amhr2 ${ }^{\text {cre/+ }}$ (solid column, $n=3$ ) mice at various ages, when the value of the P21 control was set to 100, is shown in the graph. Error bars represent the S.E.M., and an asterisk $\left(^{*}\right)$ above a given column indicates a significant difference compared with respective control value $(P<0.05)$. 

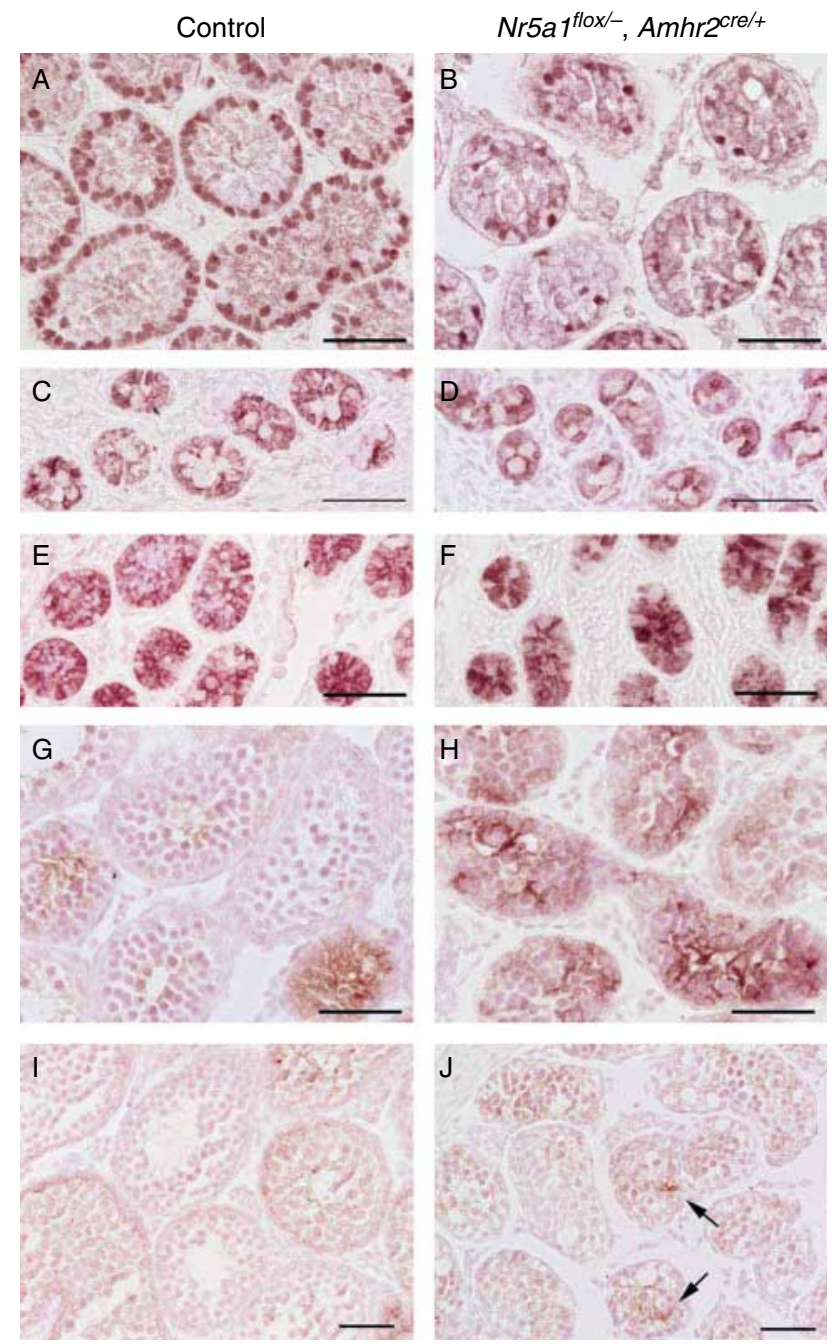

Figure 3 Impaired Sertoli cell differentiation in gonad-specific Nr5a1 $\mathrm{KO}$ testes during early postnatal development. Representative images of immunoexpression for $\mathrm{p} 27$ ( $\mathrm{A}$ and $\mathrm{B})$ and $\mathrm{AMH}(\mathrm{C}, \mathrm{D}, \mathrm{E}, \mathrm{F}, \mathrm{G}, \mathrm{H}, \mathrm{I}$ and $\mathrm{J}$ ), which are differentiated and immature Sertoli cell markers, respectively, in testes from controls (left panels) and $\mathrm{Nr} 5 \mathrm{a} \mathrm{f}^{\text {flox/- }}$,

Amhr2 ${ }^{\text {cre/+ }}$ (right panels) mice, at P0 (C and D), P7 (E and F), P14 (A, B, $\mathrm{G}$ and $\mathrm{H}$ ), and P21 (I and J). Arrows indicate sporadically intense AMHimmunoreactive spots. Scale bars $=50 \mu \mathrm{m}$.

in both $\mathrm{KO}$ and control mouse testes (Fig. 3C, D, E and F). In control testes at P14, AMH ir was reduced in most tubules, although there were some tubules with $\mathrm{AMH}$ staining that tended to be restricted to the periphery (Fig. 3G). In the $\mathrm{KO}$ mouse testes, such a centerto-periphery wave of downregulation of $\mathrm{AMH}$ was not detected, and many of the seminiferous tubules still exhibited intense $\mathrm{AMH}$ ir (Fig. $3 \mathrm{H}$ ). These results indicate that the Sertoli cells in the KO mouse testes are retained in an immature state. At P21, AMH staining was negative or very faint in most of the tubules in controls (Fig. 3I). In the $\mathrm{KO}$ mouse testes, most tubules were nearly negative for $\mathrm{AMH}$ at this point, but intense $\mathrm{AMH}$ spots were sporadically detected within some tubules (Fig. 3J).
We further examined the expression of Sertoli cell markers including WT1, SOX9, GATA4, and AR. Positive staining for all the factors was detected in Sertoli cells, and there was no clear difference between the gonadspecific Nr5a1 KO and control mouse testes at P0 or P7 (data not shown). We observed a reduction in markerpositive cells in the $\mathrm{KO}$ mouse testes compared with controls at P14 and P21, although the magnitude of the change was distinct for each of the factors (Fig. 4A, B, C, $D, E, F, G$ and $H)$. We assayed the number of Sertoli cells expressing each factor by counting positive cells. The results showed that the number of marker-positive cells was significantly lower in $\mathrm{KO}$ mouse testes compared with controls at all stages and for all markers except for AR at P0, which is not expressed in Sertoli cells (Fig. 4l, J, $\mathrm{K}$ and $\mathrm{L}$ ).

\section{Presence of Leydig cells in the gonad-specific Nr5a1 KO testis during postnatal development}

To identify any steroidogenic interstitial cells, we performed IHC for CYP11A1. CYP11A1 ir was detected in interstitial cells in both the gonad-specific Nr5a1 KO and the control mouse testes at all stages examined (Fig. 5). These results indicate the presence of steroidogenic Leydig cells in the $\mathrm{KO}$ testes throughout postnatal development.

\section{Cellular proliferation and apoptosis}

To study cell proliferation in the different cell types of control and gonad-specific Nr5a1 KO testes during postnatal development, double-label IHC for proliferating cell nuclear antigen (PCNA) and NR5A1 was performed. The expression pattern was similar between control and $\mathrm{KO}$ testes at P0 and P7 (data not shown). At P14 and P21, however, most germ cells were positive for PCNA in control testes (Fig. 6A, B, C, G and H), whereas there were a large number of PCNA-negative germ cells in the $\mathrm{KO}$ testes (Fig. 6D, E, F, J, K and L). In the interstitium, PCNA-positive Leydig cells were occasionally detected in both control and $\mathrm{KO}$ testes at P14 (Fig. 6A, B, C, D, E and F). At P21, they were rarely detected in controls but still present in $\mathrm{KO}$ testes (Fig. 6G, H, I, J, K and L).

Apoptotic cell death was examined by TUNEL assay. TUNEL-labeled cells were detected within many tubular sections of both control and the $\mathrm{KO}$ testes, but their numbers were significantly higher in the $K O$ testes compared with controls at both P14 and P21 (Fig. 7A, B, $\mathrm{C}, \mathrm{D}$ and $\mathrm{E})$. To determine whether the apoptotic cells are Sertoli cells, IHC for SOX9, a Sertoli cell marker, and the TUNEL assay were performed on adjacent serial sections of the KO testes. SOX9-positive Sertoli cells displayed irregular-shaped nuclei and were localized at the basal layer of the seminiferous tubule, whereas most 

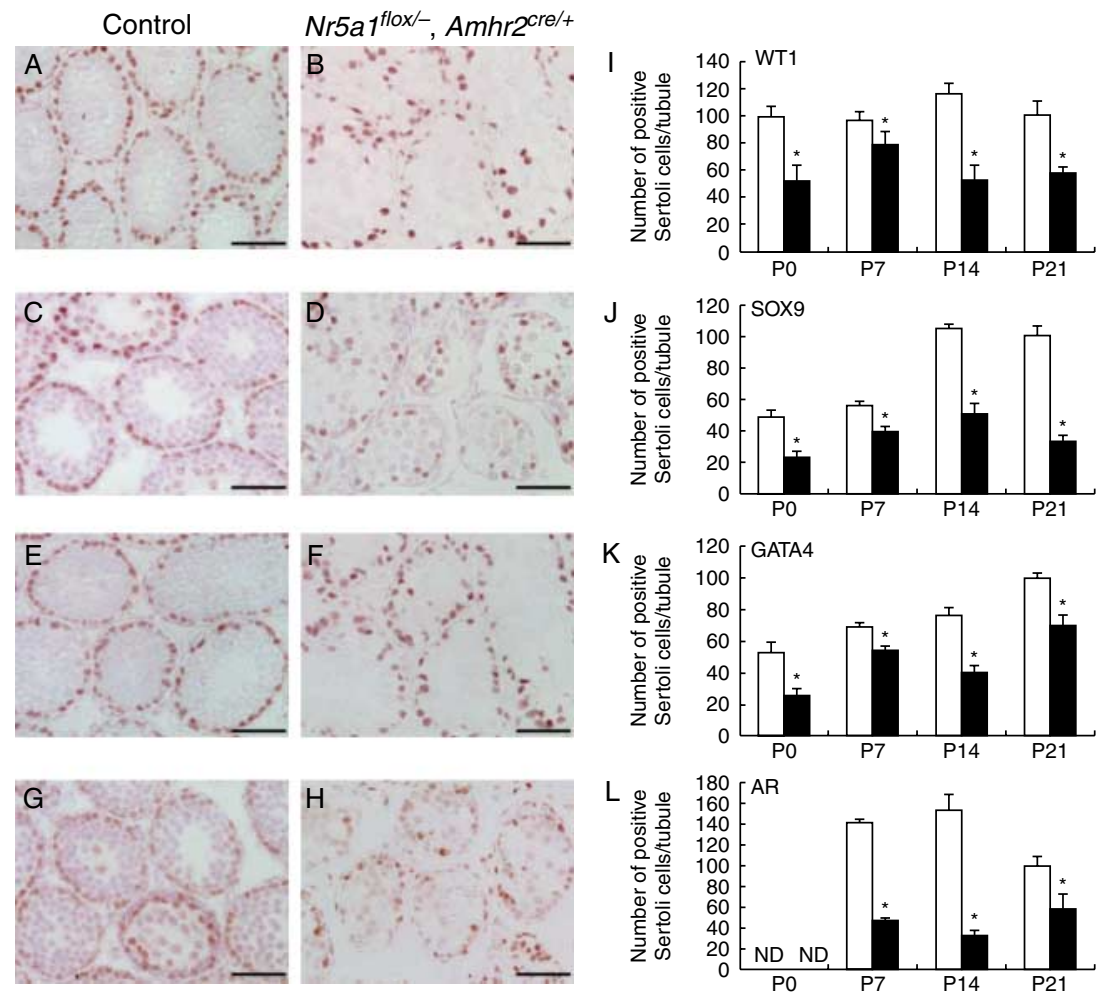

Figure 4 Expression of Sertoli cell markers in gonad-specific Nr5a1 KO testes. (A, B, C, D, E, F, G and H) Representative images of immunoexpression for the Sertoli cell markers WT1 (A and B), SOX9 (C and D), GATA4 (E and F), and AR (G and H) in testes from controls (left panels) and Nr5a ${ }^{f l o x /-}$, Amhr2 ${ }^{\text {cre/+ }}$ (right panels) mice at P21. Scale bars $=50 \mu \mathrm{m}$. (I, J, K and L) Sertoli cells immunoreactive for WT1, SOX9, GATA4, or AR per tubule were counted. The relative number of control (open column, $n=3$ ) and $\mathrm{Nr}_{5} \mathrm{a}^{\text {flox } /-}, A m h r 2^{\text {cre/ }+}$ (solid column, $n=3$ ) mice at various ages, when the value of the P21 control was set to 100, was shown in the graph. Error bars represent the s.E.M., and an asterisk $(*)$ above a given column indicates a significant difference compared with respective control value $(P<0.05)$. ND, not done.

TUNEL-labeled nuclei were round and detected through the whole depth of the tubular epithelium, indicating that most TUNEL-labeled cells are SOX9 negative (Fig. 7F, G, H and I).

\section{Discussion}

As Amhr2-Cre is expressed in both Leydig and Sertoli cells in the adult mouse testes (Jeyasuria et al. 2004, Boyer et al. 2008), both cell types can be targeted in Amhr2-Cre-mediated KO testes. Interestingly, this study demonstrates that in the gonad-specific Nr5a1 KO testes, the number of Sertoli cells appears to be lower than controls, while the Leydig cells remained at all the developmental stages. These results were unexpected because Sertoli cells but not Leydig cells were detected in $\mathrm{KO}$ testes at E14 and E16 in a previous study (Jeyasuria et al. 2004). Several studies on conditional KO mice generated with Amhr2-Cre have reported complexities in the phenotype. For example, both Leydig and Sertoli cells were affected in $\mathrm{Ar} \mathrm{KO}$ testes generated using Amhr2-Cre (Xu et al. 2007), and Amhr2-Cre-mediated Gata4 KO mice displayed Sertoli cell-specific ablation of the gene in postnatal testes (Kyrönlahti et al. 2011). As suggested in those reports, one possible explanation is inefficient Cre-mediated recombination in Leydig cells. Alternatively, developmentally different phenotypes may be attributed to the complex Amhr2 expression profile during testicular development. In situ hybridization results for 21-day-old rat testes have demonstrated that Amhr2 mRNA expression is found in seminiferous tubules but not in the interstitium (Baarends et al. 1995). More recently, expression analysis of a floxed YFP reporter driven by Amhr2-Cre demonstrated that all Sertoli cells were YFP positive in the adult testes, but few YFP-positive Sertoli cells were found in embryonic and neonatal testes (Tanwar et al. 2010). These reports indicate spatial and temporal changes in the expression of Amhr2 during testicular development.

This study showed that $\mathrm{Nr} 5 \mathrm{a} 1$ expression in Sertoli cells progressively decreased during postnatal development and it had almost completely disappeared by P21 in the gonad-specific Nr5a1 KO mice. Despite the finding that relative numbers of NR5A1-positive Sertoli cells in the $\mathrm{KO}$ testis were significantly lower compared with the control testis as early as P0, the overall architecture of the seminiferous tubules did not appear to be histologically different from control testes of littermates from P0 until P7. During the period from P0 to P14, the number of NR5A1-positive Sertoli cells in 

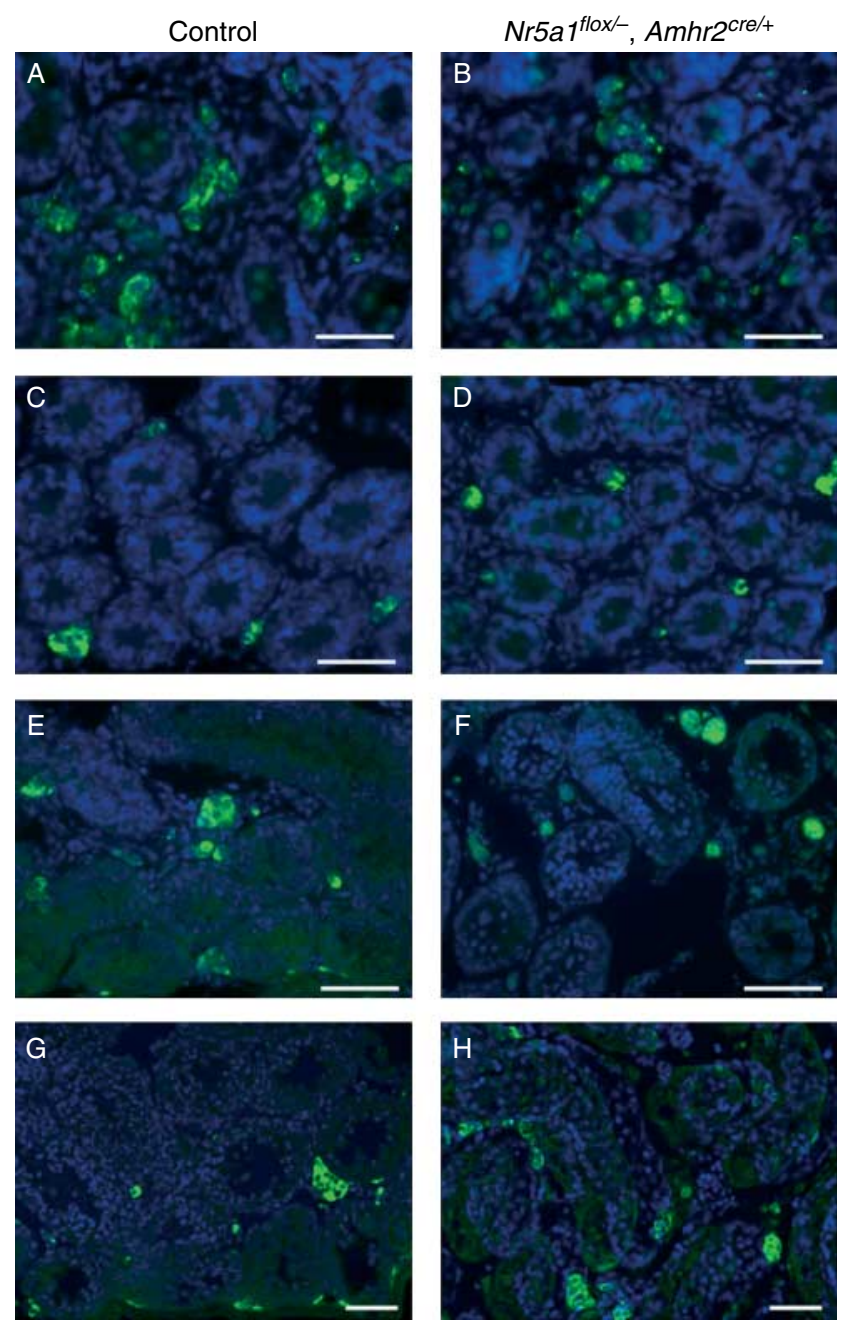

Figure 5 Localization of steroidogenic cells in gonad-specific $\mathrm{Nr} 5 \mathrm{a} 1 \mathrm{KO}$ testes during early postnatal development. Sections of testes from controls (left panels) and $\mathrm{Nr} 5 \mathrm{a} 1^{\text {flox/- }}$, Amhr2 ${ }^{\text {cre/+ }}$ (right panels) mice were subjected to immunofluorescence for CYP11A1 (green), a marker for steroidogenic Leydig cells, and nuclear-stained with DAPI (blue). (A and B) P0. (C and D) P7. (E and F) P14. (G and H) P21. Scale bars $=50 \mu \mathrm{m}$.

the $\mathrm{KO}$ testis remained similar, while it progressively increased in the control testis. Despite a partial depletion of Sertoli cells as early as P0, the overall architecture of the seminiferous tubules in the gonad-specific Nr5a1 KO testes appeared grossly normal until P7. This may indicate that the few Sertoli cells remaining in the KO testis can sufficiently maintain seminiferous tubule structure up to P7. Abnormal seminiferous tubular architecture accompanied with irregularly arranged germ cells, vacuoles, and giant cells in the gonadspecific Nr5a1 KO testes became apparent by P14. Intratubular vacuoles have been reported in other $\mathrm{KO}$ mice, in which spermatogenesis was impaired and is associated with Sertoli cell damage (Hellsten et al. 2002, Kyrönlahti et al. 2011). In fact, we observed that many of the vacuoles detected in the $\mathrm{KO}$ mice were accompanied by a nucleus positive for the Sertoli cell marker
SOX9 (Fig. 7l). The giant cells detected in this study morphologically resemble those named meiotic micronucleate giant spermatocytes (MMGs), which contain disaggregated bivalent or replicated univalent chromosomes due to incomplete meiotic division of metaphase spermatocytes (Faisal et al. 2008). MMGs can be induced by aneuploidogenic agents including aflatoxins, which are known to cause disruption of testicular histoarchitecture and spermatogenesis. Thus, all the morphological abnormalities seem to be associated with impaired Sertoli cell development.

Sertoli cells are known to play a central role in regulating early postnatal development of the testis (Nel-Themaat et al. 2011). We detected no apparent differences in Amh expression between $\mathrm{KO}$ and control testes at P0 and P7. As Amh is a direct target gene of NR5A1 regulation, these results indicate that NR5A1positive Sertoli cells in the $\mathrm{KO}$ testis can produce $\mathrm{AMH}$ at levels comparable with those in normal testes at these time points. At P14, a higher number of intensely AMHpositive tubules concomitant with a lower number of p27-positive Sertoli cells were seen in the KO testes suggesting a delay of Sertoli cell differentiation. However, at P21, Amh expression was negative in most tubules except for a few tubules with sporadic $\mathrm{AMH}$-producing cells in $\mathrm{KO}$ testes. This diminished Amh expression is likely due to the deletion of NR5A1 in the Sertoli cells.

Despite nearly complete loss of NR5A1 expression at P21, expression of SOX9, WT1, and GATA4 was preserved in Sertoli cells to some extent, suggesting that these proteins may interact indirectly with NR5A 1 in Sertoli cells in postnatal testes. Nevertheless, the organization of the seminiferous tubules was severely disrupted in $\mathrm{KO}$ mice. Therefore, tubular disorganization is likely to be primarily due to the loss of NR5A1, suggesting an essential role of NR5A1 in Sertoli cells in the development of seminiferous tubules.

Both active proliferation and apoptosis occur during the first wave of spermatogenesis at 2-3 weeks after birth (Rodriguez et al. 1997). We demonstrated a marked reduction of PCNA-positive germ cells in the $\mathrm{KO}$ testes at P14 and P21. Further, our TUNEL assay showed a significant increase in apoptosis levels in $\mathrm{KO}$ testes. Based on the morphology and localization of SOX9positive and TUNEL-labeled cells, most of the apoptotic cells detected in $\mathrm{KO}$ testes are presumptively germ cells (SOX9 negative), although it is still possible that some of the TUNEL-labeled nuclei that were located near the base of the seminiferous tubules are degenerating Sertoli cells. Taken together, these results indicate that both cellular proliferation and apoptosis are affected in seminiferous tubules of the gonad-specific Nr5a1 $\mathrm{KO}$ testes.

It is well known that the androgens, primarily testosterone, are required for normal testicular development and spermatogenesis (Holdcraft \& Braun 2004). 

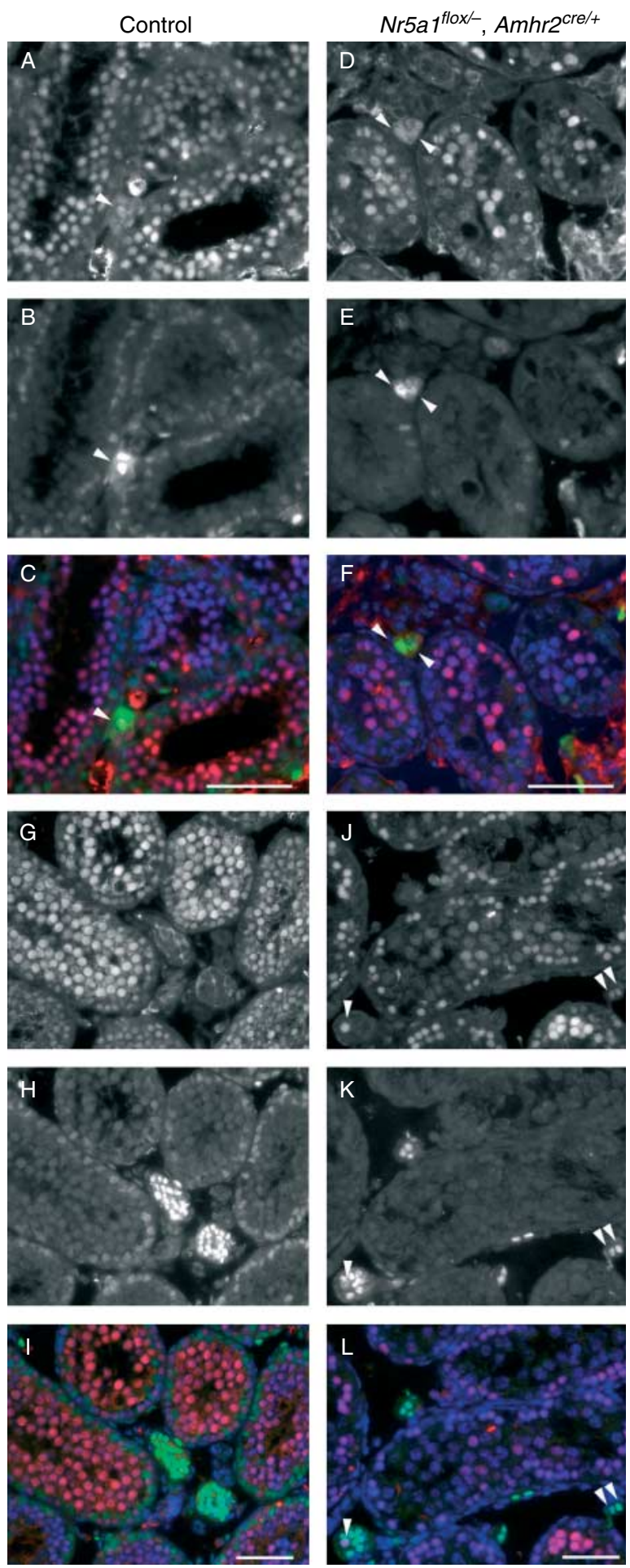

Figure 6 Localization of proliferating cells in gonad-specific $\mathrm{Nr} 5 \mathrm{a} 1 \mathrm{KO}$ testes. Sections of testes from controls (left panels) and $\mathrm{Nr}^{2} \mathrm{a} 7^{\text {flox/- }}$, Amhr ${ }^{\text {cre/+ }}$ (right panels) mice were subjected to double-label immunofluorescence for PCNA (red) and NR5A1 (green) and were nuclear stained with DAPI (blue). (A, B, C, D, E and F) P14. (G, H, I, J, K and $\mathrm{L}) \mathrm{P} 21$. Gray-scale images for PCNA (A, D, G and J) and NR5A1 (B, $\mathrm{E}, \mathrm{H}$ and $\mathrm{K})$ and merged color images $(\mathrm{C}, \mathrm{F}, \mathrm{I}$ and $\mathrm{L})$ are presented. Arrowheads indicate double-labeled Leydig cells. Scale bars $=50 \mu \mathrm{m}$.
We did not measure either plasma or intratesticular testosterone levels in this study. However, we demonstrated that CYP11A1-positive cells were present in the interstitium of the gonad-specific Nr5a1 KO testes at all the developmental stages. Also, androgen-dependent internal and external genital organs were formed in the Nr5a1 KO male mice, although they appeared slightly smaller than normal (data not shown). As Leydig cells are a primary source for testosterone in postnatal testes, these findings strongly suggest a presence of Leydig cells that produce testosterone in $\mathrm{KO}$ testes throughout postnatal development. However, we found an increase in PCNA-positive Leydig cells in the KO testes at P21, suggesting that Leydig cell proliferation is dysregulated. Therefore, it might be possible that spermatogenesis is influenced indirectly by abnormal Leydig cells, as Leydig cells produce not only testosterone but also other hormones including growth factors, cytokines, and vasoactive peptides, which contribute to the intercellular communication between Leydig and Sertoli cells.

In summary, we have described the postnatal development of the gonad-specific Nr5a1 KO mouse testes from birth into the prepubertal stages and have shown several abnormal features of testicular development, leading to infertility in adulthood. Although further studies are required to elucidate the precise postnatal physiological functions of NR5A1, our findings provide in vivo evidence that NR5A1 is essential for Sertoli cell function and spermatogenesis in the testis.

\section{Materials and Methods}

\section{Experimental mice}

Procedures involving mice were approved by the Institutional Animal Care and Use Committee at Yokohama City University School of Medicine. Amhr2 ${ }^{\text {cre/+ }}$ mice (also termed B6; 129S7$\left.\mathrm{Amhr} 2^{\mathrm{tm} 3(\mathrm{cre}) \mathrm{Bhr}} / \mathrm{Mmnc}\right)$ were obtained from the MMRRC (Chapel Hill, NC, USA) and genotyped as described elsewhere (Jeyasuria et al. 2004). We inactivated Nr5a1 using the Amhr2Cre mouse strain (Jamin et al. 2002). To generate conditional $\mathrm{KO}$ mice, $\mathrm{Nr}^{2} \mathrm{a}^{+/-}, \mathrm{Amhr}^{\mathrm{cre} /+}$ mice were mated to $\mathrm{Nr} 5 \mathrm{a} 1^{\text {flox flox }}$ mice and all four genotypes were recovered at the expected Mendelian frequency. We conducted a timecourse analysis of postnatal testis development in $\mathrm{Nr} 5 \mathrm{a} \mathrm{f}^{\text {flox/- }}$, Amhr2 ${ }^{\text {cre/ }+}$ conditional KO pups. All mice had free access to water and standard rodent chow and were exposed to $14 \mathrm{~h}$ light: $10 \mathrm{~h}$ darkness photoperiods. Mice were genotyped by PCR assays using primer sets (Jeyasuria et al. 2004) and were killed at P0, P7, P14, and P21 by cervical dislocation. The day of birth was considered $\mathrm{P} 0$.

\section{Histological and immunohistochemical analyses}

Testes harvested from pups were fixed overnight in $4 \%(\mathrm{w} / \mathrm{v})$ paraformaldehyde in PBS and embedded in paraffin. Paraffin sections ( $6 \mu \mathrm{m}$ thickness) were deparaffinized, rehydrated, and stained with hematoxylin and eosin for histological analysis. 

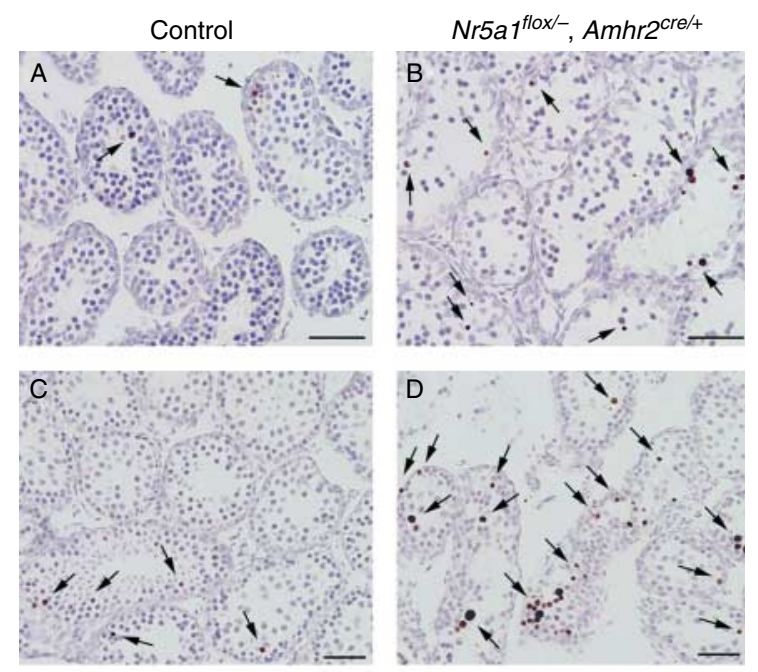

E
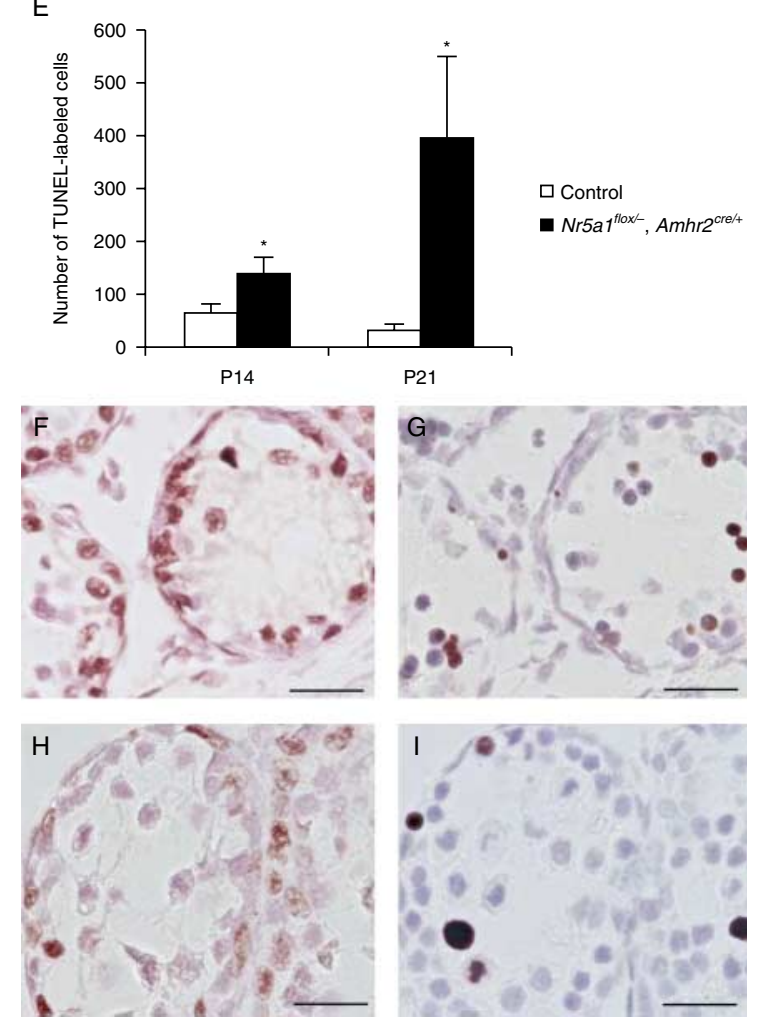

Figure 7 Localization of apoptotic cells in gonad-specific Nr5a1 KO testes. (A, B, C and D) Sections of testes from controls (left panels) and $\mathrm{Nr} 5 \mathrm{a} 7^{\text {flox } /-}$, Amhr2 ${ }^{\text {cre/+ }}$ (right panels) mice were subjected to TUNEL assay. (A and B) P14. (C and D) P21. Arrows indicate representative TUNEL-labeled nuclei (brown spots). (E) TUNEL-labeled cells were counted in the square field $\left(1 \mu \mathrm{m}^{2}\right)$ and were shown as the total count of six fields from the testis sections of three mice per each group. Open and solid columns represent the TUNEL-labeled cell number in the control and $\mathrm{Nr} 5 \mathrm{ar} 1^{\text {flox/- }}$, Amhr $2^{\text {cre/+ }}$ testes, respectively, at P14 and P21 $\left(n=3\right.$ for each column). Error bars represent the S.E.M. and an asterisk $\left(^{*}\right)$ above a given column indicates a significant difference compared with respective control value $(P<0.05)$. $(\mathrm{F}, \mathrm{G}, \mathrm{H}$ and $\mathrm{I})$ Representative images of immunoexpression for SOX9 $(\mathrm{F}$ and $\mathrm{H})$ and TUNEL staining $(\mathrm{G}$ and $\mathrm{I})$ in adjacent serial sections of $\mathrm{KO}$ testes at $\mathrm{P} 14$ ( $\mathrm{F}$ and $\mathrm{G}$ ) and $\mathrm{P} 21$ ( $\mathrm{H}$ and $\mathrm{I}$ ). Scale bars $=50 \mu \mathrm{m}$ in $\mathrm{A}, \mathrm{B}, \mathrm{C}$ and $\mathrm{D}, 25 \mu \mathrm{m}$ in $\mathrm{F}, \mathrm{G}, \mathrm{H}$ and $\mathrm{I}$.
For IHC and immunofluorescence, antigen retrieval was performed by microwave-boiling sections for $5 \mathrm{~min}$ in $0.01 \mathrm{M}$ citrate buffer $(\mathrm{pH}$ 6.0). Endogenous peroxidase activity was quenched by immersing sections in 3\% (v/v) hydrogen peroxide in PBS for 15 min. After three washes in PBS, the sections were incubated in a blocking solution, $5 \%(\mathrm{w} / \mathrm{v})$ Block Ace (Dainippon Pharmaceutical Co., Ltd., Tokyo, Japan), at room temperature for $30 \mathrm{~min}$ and subsequently incubated with a diluted primary antibody overnight at $4{ }^{\circ} \mathrm{C}$. Rabbit anti-NR5A1 antibody (lkeda et al. 2001; 1:10 000 dilution), goat anti-AMH antibody (sc-6886; 1:5000; Santa Cruz Biotechnology, Inc., Santa Cruz, CA, USA), rabbit anti-p27 antibody (sc-528; 1:2000; Santa Cruz), rabbit antiWT1 antibody (sc-192; 1:50 000; Santa Cruz), rabbit antiSOX9 antibody (sc-2095; 1:500; Santa Cruz), goat anti-GATA4 antibody (sc-1237; 1:2000; Santa Cruz), and rabbit anti-AR antibody (PA1-111A; 1:2000; Thermo Fisher Scientific, Inc., Rockford, IL USA) were used as primary antibodies. After washing with $\mathrm{PBS}+0.1 \%(\mathrm{v} / \mathrm{v})$ Triton $\mathrm{X}$, sections were incubated with either N-Histofine Simple Stain Mouse MAX PO (R) (Nichirei Biosciences, Tokyo, Japan) or N-Histofine Simple Stain Mouse MAX PO (G) (Nichirei). The peroxidase reaction was visualized using Vector NovaRED (Vector Laboratories, Burlingame, CA, USA). Slides were dehydrated and mounted with Mount-Quick (Daido Sangyo, Saitama, Japan). For immunofluorescence, after incubation with the rabbit anti-CYP11A1 antibody (AB1244, 1:5000; Chemicon International, Inc., Temecula, CA, USA), the sections were rinsed in PBS and incubated in secondary antibody conjugated with Alexa Fluor 488 (1:500; Molecular Probes/Invitrogen, Carlsbad, CA, USA) for $2 \mathrm{~h}$ at room temperature. Sections were rinsed in PBS, counterstained with 4,6-diamidino-2-phenylindole (DAPI, 1:1000; Sigma-Aldrich) to stain the nucleus, and mounted using Fluoromount (Diagnostic BioSystems, Pleasanton, CA, USA). On each occasion, sections from at least three testes in each genotype group at each time point were processed in parallel for comparison of immunostaining on at least two separate occasions to ensure reproducibility of results. For negative controls, tissue sections were incubated without addition of primary antibody. Digital images were taken using a Keyence BIOREVO microscope (BZ-9000) and were transferred to Photoshop CS for the generation of figures.

\section{Counting NR5A1-, WT1-, SOX9-, GATA4-, and AR-positive Sertoli cells}

After testicular sections were immunostained for NR5A1, WT1, SOX9, GATA4, and AR as described above, the number of immunoreactive Sertoli cells per seminiferous tubule was determined by counting 10-15 tubules of each genotype at each developmental stage. Only round tubules were counted. Three testicular sections from three animals for each time point and genotype were analyzed.

\section{Double-label immunofluorescence}

Colocalization of NR5A1 and PCNA was examined by doublelabel immunofluorescence. Sections were coincubated overnight at $4{ }^{\circ} \mathrm{C}$ with rabbit anti-NR5A1 and mouse anti-PCNA 
(1:50; Zymed, San Francisco, CA, USA). After incubation with the primary antibodies, the sections were rinsed in PBS and incubated in secondary antibodies conjugated with Alexa Fluor 488 or 546 (1:500; Molecular Probes/Invitrogen) for $2 \mathrm{~h}$ at room temperature. Sections were rinsed in PBS, incubated with DAPI to stain the nucleus, and mounted using Fluoromount. Digital images were taken using a BZ-9000 microscope and were superimposed and processed with Adobe Photoshop CS.

\section{TUNEL assay}

After deparaffinization, the tissue sections were processed for TUNEL staining using an in situ apoptosis detection kit (TaKaRa Bio, Shiga, Japan). FITC-conjugated dCTPs were incorporated into nick DNA for $90 \mathrm{~min}$ at $37^{\circ} \mathrm{C}$ in the presence of terminal deoxynucleotidyl transferase. The sections were rinsed in PBS and incubated with HRP-conjugated anti-FITC antibody for $30 \mathrm{~min}$ at $37^{\circ} \mathrm{C}$. The peroxidase reaction was visualized using Vector NovaRED (Vector). The sections were lightly counterstained with hematoxylin. Slides were dehydrated and mounted with Mount-Quick (Daido Sangyo). Six fields, each of which was defined by a square field $\left(1 \mu \mathrm{m}^{2}\right)$ in the image of the Keyence BIOREVO microscope (BZ-9000), were randomly selected from the testis sections of three mice per each group. The total count of TUNEL-positive cells from the six fields was shown in the graph (Fig. 7E). SOX9 IHC and TUNEL-staining were performed on the adjacent serial sections of P14 and P21 $\mathrm{KO}$ mouse testes.

\section{Statistical analysis}

Statistical analysis was performed using Microsoft Excel 97 SR-2 (Microsoft Corporation). At each developmental stage, the significance of differences in labeled cell number between the control and the $\mathrm{KO}$ groups was evaluated using one-way ANOVA $(P<0.05)$.

\section{Declaration of interest}

The authors declare that there is no conflict of interest that could be perceived as prejudicing the impartiality of the research reported.

\section{Funding}

This work was supported by a grant-in-aid for scientific research from the Japan Society for the Promotion of Science to Y Ikeda (grant number: 21590219).

\section{Acknowledgements}

The authors would like to thank Masahito Takiguchi for valuable technical assistance.

\section{References}

Arango NA, Lovell-Badge R \& Behringer RR 1999 Targeted mutagenesis of the endogenous mouse Mis gene promoter: in vivo definition of genetic pathways of vertebrate sexual development. Cell 99 409-419. (doi:10.1016/S0092-8674(00)81527-5)

Baarends WM, Hoogerbrugge JW, Post M, Visser JA, De Rooij D, Parvinen M, Themmen AP \& Grootegoed JA 1995 Anti-Müllerian hormone and anti-Müllerian hormone type II receptor messenger ribonucleic acid expression during postnatal testis development and in the adult testis of the rat. Endocrinology 136 5614-5622. (doi:10.1210/ en.136.12.5614)

Beumer TL, Kiyokawa H, Roepers-Gajadien HL, van den Bos LA, Lock TM, Gademan IS, Rutgers DH, Koff A \& de Rooij DG 1999 Regulatory role of p27kip1 in the mouse and human testis. Endocrinology $1401834-1840$. (doi:10.1210/en.140.4.1834)

Boyer A, Hermo L, Paquet M, Robaire B \& Boerboom D 2008 Seminiferous tubule degeneration and infertility in mice with sustained activation of WNT/CTNNB1 signaling in sertoli cells. Biology of Reproduction 79 475-485. (doi:10.1095/biolreprod.108.068627)

De Santa Barbara P, Bonneaud N, Boizet B, Desclozeaux M, Moniot B, Sudbeck P, Scherer G, Poulat F \& Berta P 1998 Direct interaction of SRY-related protein SOX9 and steroidogenic factor 1 regulates transcription of the human anti-Müllerian hormone gene. Molecular and Cellular Biology 18 6653-6665.

Faisal K, Faridha A \& Akbarsha MA 2008 Induction of meiotic micronuclei in spermatocytes in vivo by aflatoxin B1: light and transmission electron microscopic study in Swiss mouse. Reproductive Toxicology 26 303-309. (doi:10.1016/j.reprotox.2008.09.003)

Hellsten E, Bernard DJ, Owens JW, Eckhaus M, Suchy SF \& Nussbaum RL 2002 Sertoli cell vacuolization and abnormal germ cell adhesion in mice deficient in an inositol polyphosphate 5-phosphatase. Biology of Reproduction 66 1522-1530. (doi:10.1095/biolreprod66.5. 1522)

Holdcraft RW \& Braun RE 2004 Hormonal regulation of spermatogenesis. International Journal of Andrology 27 335-342. (doi:10.1111/j.13652605.2004.00502.x)

Ikeda Y, Lala DS, Luo X, Kim E, Moisan MP \& Parker KL 1993 Characterization of the mouse FTZ-F1 gene, which encodes a key regulator of steroid hydroxylase gene expression. Molecular Endocrinology 7 852-860. (doi:10.1210/me.7.7.852)

Ikeda Y, Shen WH, Ingraham HA \& Parker KL 1994 Developmental expression of mouse steroidogenic factor-1, an essential regulator of the steroid hydroxylases. Molecular Endocrinology 8 654-662. (doi:10.1210/me.8.5.654)

Ikeda Y, Luo X, Abbud R, Nilson JH \& Parker KL 1995 The nuclear receptor steroidogenic factor 1 is essential for the formation of the ventromedial hypothalamic nucleus. Molecular Endocrinology 9 478-486. (doi:10.1210/me.9.4.478)

Ikeda Y, Nagai A, Ikeda M \& Hayashi S 2001 Neonatal estrogen exposure inhibits steroidogenesis in the developing rat ovary. Developmental Dynamics 221 443-453. (doi:10.1002/dvdy.1162)

Jamin SP, Arango NA, Mishina Y, Hanks MC \& Behringer RR 2002 Requirement of Bmpr1a for Müllerian duct regression during male sexual development. Nature Genetics 32 408-410. (doi:10.1038/ ng1003)

Jeyasuria P, Ikeda Y, Jamin SP, Zhao L, De Rooij DG, Themmen AP, Behringer RR \& Parker KL 2004 Cell-specific knockout of steroidogenic factor 1 reveals its essential roles in gonadal function. Molecular Endocrinology 18 1610-1619. (doi:10.1210/me.2003-0404)

Kyrönlahti A, Euler R, Bielinska M, Schoeller EL, Moley KH, Toppari J, Heikinheimo M \& Wilson DB 2011 GATA4 regulates Sertoli cell function and fertility in adult male mice. Molecular and Cellular Endocrinology 333 85-95. (doi:10.1016/j.mce.2010.12.019)

Luo X, Ikeda Y \& Parker KL 1994 A cell-specific nuclear receptor is essential for adrenal and gonadal development and sexual differentiation. Cell 77 481-490. (doi:10.1016/0092-8674(94)90211-9)

Nachtigal MW, Hirokawa Y, Enyeart-VanHouten DL, Flanagan JN, Hammer GD \& Ingraham HA 1998 Wilms' tumor 1 and Dax-1 modulate the orphan nuclear receptor SF-1 in sex-specific gene expression. Cel/ 93 445-454. (doi:10.1016/S0092-8674(00)81172-1) 
Nel-Themaat L, Jang CW, Stewart MD, Akiyama H, Viger RS \& Behringer RR 2011 Sertoli cell behaviors in developing testis cords and postnatal seminiferous tubules of the mouse. Biology of Reproduction $\mathbf{8 4}$ 342-350. (doi:10.1095/biolreprod.110.086900)

Parker KL, Rice DA, Lala DS, Ikeda Y, Luo X, Wong M, Bakke M, Zhao L, Frigeri C, Hanley NA et al. 2002 Steroidogenic factor 1: an essential mediator of endocrine development. Recent Progress in Hormone Research 57 19-36. (doi:10.1210/rp.57.1.19)

Pelusi C, Ikeda Y, Zubair M \& Parker KL 2008 Impaired follicle development and infertility in female mice lacking steroidogenic factor 1 in ovarian granulosa cells. Biology of Reproduction 79 1074-1083. (doi:10.1095/biolreprod.108.069435)

Rey R 1998 Endocrine, paracrine and cellular regulation of postnatal antiMullerian hormone secretion by Sertoli cells. Trends in Endocrinology and Metabolism 9 271-276. (doi:10.1016/S1043-2760(98)00069-1)

Rodriguez I, Ody C, Araki K, Garcia I \& Vassalli P 1997 An early and massive wave of germinal cell apoptosis is required for the development of functional spermatogenesis. EMBO Journal 16 2262-2270. (doi:10. 1093/emboj/16.9.2262)

Schimmer BP \& White PC 2010 Minireview: steroidogenic factor 1: its roles in differentiation, development, and disease. Molecular Endocrinology 24 1322-1337. (doi:10.1210/me.2009-0519)

Sharpe RM, McKinnell C, Kivlin C \& Fisher JS 2003 Proliferation and functional maturation of Sertoli cells, and their relevance to disorders of testis function in adulthood. Reproduction 125 769-784. (doi:10.1530/ rep.0.1250769)
Shen WH, Moore CC, Ikeda Y, Parker KL \& Ingraham HA 1994 Nuclear receptor steroidogenic factor 1 regulates the mullerian inhibiting substance gene: a link to the sex determination cascade. Cell 77 651-661. (doi:10.1016/0092-8674(94)90050-7)

Tanwar PS, Kaneko-Tarui T, Zhang L, Rani P, Taketo MM \& Teixeira J 2010 Constitutive WNT/beta-catenin signaling in murine Sertoli cells disrupts their differentiation and ability to support spermatogenesis. Biology of Reproduction 82 422-432. (doi:10.1095/biolreprod.109.079335)

Watanabe K, Clarke TR, Lane AH, Wang X \& Donahoe PK 2000 Endogenous expression of Müllerian inhibiting substance in early postnatal rat sertoli cells requires multiple steroidogenic factor- 1 and GATA-4-binding sites. PNAS 97 1624-1629. (doi:10.1073/pnas.97.4. 1624)

Xu Q, Lin HY, Yeh SD, Yu IC, Wang RS, Chen YT, Zhang C, Altuwaijri S, Chen LM, Chuang KH et al. 2007 Infertility with defective spermatogenesis and steroidogenesis in male mice lacking androgen receptor in Leydig cells. Endocrine 32 96-106. (doi:10.1007/s12020007-9015-0)

Received 29 September 2011

First decision 31 October 2011

Revised manuscript received 20 February 2012

Accepted 12 March 2012 